\title{
Young-onset breast cancer: a poor prognosis only exists in low-risk patients
}

\author{
Jianfei $\mathrm{Fu}^{1 *}$, Lunpo $\mathrm{Wu}^{2,3^{*}}$, Tiantian $\mathrm{Xu}^{4}$, Dan $\mathrm{Li}^{5}, 6$, Mingliang Ying7, Mengjie Jiang 8 , Ting Jiang 9 , Wei Fu10, \\ Fan Wang ${ }^{11}$, Jinlin $\mathrm{Du}^{12}$ \\ 1. Department of Medical Oncology, Jinhua Hospital, Zhejiang University School of Medicine, Jinhua 321000, Zhejiang Province, China. \\ 2. Department of Gastroenterology, Second Affiliated Hospital of Zhejiang University School of Medicine, Hangzhou 310009, Zhejiang Province, China. \\ 3. Institute of Gastroenterology, Zhejiang University, Hangzhou 310009, Zhejiang Province, China. \\ 4. Institute of Translational Medicine, School of Medicine, Zhejiang University, Hangzhou 310029, Zhejiang Province, China. \\ 5. Cancer Institute (Key Laboratory of Cancer Prevention and Intervention, Chinese National Ministry of Education; Key Laboratory of Molecular Biology in \\ Medical Sciences, Zhejiang Province, China), Second Affiliated Hospital of Zhejiang University School of Medicine, Hangzhou 310009, Zhejiang Province, \\ China. \\ 6. Department of Medical Oncology, Second Affiliated Hospital of Zhejiang University School of Medicine, Hangzhou 310009, Zhejiang Province, China. \\ 7. Department of Radiology, Jinhua Hospital, Zhejiang University School of Medicine, Jinhua 321000, Zhejiang Province, China \\ 8. Department of Radiation Oncology. The First Affiliated Hospital of Zhejiang Chinese Medical University, Zhejiang Provincial Hospital of TCM, Hangzhou, \\ Zhejiang Province, China. \\ 9. Department of Nuclear Medicine, Jinhua Hospital, Zhejiang University School of Medicine, Jinhua 321000, Zhejiang Province, China. \\ 10. Division of Oncology, Johns Hopkins University School of Medicine, Baltimore, Maryland, United States of America. \\ 11. Department of Oncology, Jinhua Hospital, Zhejiang University School of Medicine, Jinhua 321000, Zhejiang Province, China. \\ 12. Department of Colorectal Surgery, Jinhua Hospital, Zhejiang University School of Medicine, Jinhua 321000, Zhejiang Province, China.
}

* Jianfei Fu and Lunpo Wu are co-first authors; they have contributed equally to the work.

$\square$ Corresponding author: Jinlin Du, Professor, Department of Colorectal Surgery, Zhejiang University Jinhua Hospital, 351 Mingyue Road, Jinhua 321000 , Zhejiang Province, China. Email: dj19090@163.com; Telephone: +86-13957998111; Fax: +86-579-82552856

(c) Ivyspring International Publisher. This is an open access article distributed under the terms of the Creative Commons Attribution (CC BY-NC) license (https://creativecommons.org/licenses/by-nc/4.0/). See http://ivyspring.com/terms for full terms and conditions.

Received: 2018.10.05; Accepted: 2019.04.13; Published: 2019.06.02

\begin{abstract}
The number of reported young breast cancer cases has increased dramatically recently. The impact of age on the outcomes of breast cancers remains controversial. Our study aimed to explore the factors that can stratify the impact of young age on the prognosis of early breast cancer patients. In total, 244,324 patients with early breast cancer in the Surveillance, Epidemiology, and End Results database were identified from 1990 to 2007. Survival curves were generated using the Kaplan-Meier method. The 5- and 10-year cancer-specific survival (CSS) rates were calculated using the Life-Table method. Multivariable analyses were used to identify prognosti c variables (without age) to construct the nomograms. The risk score developed from the nomogram was used to classify the cohort into three subgroups (low-, medium- and high-risk subgroup). Approximately $8.89 \%$ of women were diagnosed with breast cancer at a young age ( $\leq 40$ years). Clinical nomogram had the potential ability to predict CSS accurately with a well C-index (0.785). Subgroup analysis indicated that the risk score as the sole factor can stratify the impact of young age on the prognosis of early breast cancer patients. Young breast cancer patients had a worse prognosis in the low-risk $(\mathrm{HR}=0.61 ; 95 \% \mathrm{Cl}: 0.57-0.65 ; P<0.001)$ or medium-risk subgroup $(\mathrm{HR}=0.89 ; 95 \% \mathrm{Cl}$ : $0.85-0.93 ; P<0.01)$ than in the high-risk subgroup $(P=0.431)$. In conclusion, the worse prognosis of young women only appeared in the low- and medium-risk subgroups rather than in the high-risk subgroup. The risk score yielded from the nomogram model can assist clinical decision making for young breast cancer patients.
\end{abstract}

Key words: Breast cancer, Survival, Age, Nomogram model, Risk score

\section{Introduction}

Breast cancer is the greatest threat to women's health and used to appear mostly in old and middle-aged women. However, a dramatic increase in the number of young breast cancer cases has been reported. Breast cancer is the leading cause of cancer death in young women [1]. The impact of age on the outcomes of patients with early breast cancer has been increasing controversial. The factor of young age, routinely considered as an independent prognostic factor in breast cancer, was incorporated into 
treatment guidelines in the past $[2,3]$. Subsequently, guidelines specifically for young breast cancer cases were enacted [4, 5]. DNA microarray technology enabled better understanding of the biological behaviour of breast cancer, and the intrinsic subtype was widely applied in clinical practice. Several data, namely, the lower incidence of Luminal A-subtype tumors and higher incidence of human epidermal grow factor receptor 2 (HER2) over-expressing subtype tumors in young breast cancer, have led to questions regarding whether the poor prognosis resulted from disparities of the molecular subtype rather than the factor of age[6-11].

However, whether the prognostic significance of age persists in resectable breast cancer even when stratified by intrinsic subtype remains controversial. Nearly 50 genes have been identified to be related to young breast cancer in the Azime et al. study. They found that young breast cancer showed higher expression of RANK-ligand, mammary stem cell and luminal progenitors and BRCA (breast cancer susceptibility gene)-1 mutation signatures beyond subtype distribution [12]. With the rapid development of precision medicine, gene signatures, such as MammaPrint[13] and Oncotype DX Recurrence Score[14], are widely used in clinical practice. There are age-specific genes when younger women $(\leq 45$ years) are compared with older women (> 65 years) [15]. However, their late study denied their previous conclusion after stratification by intrinsic subtype and proposed that the so-called age specific genes of young breast cancer function as a surrogate for aggressive intrinsic subtypes rather than demonstrate a unique biology [16]. Recently, the National Comprehensive Cancer Network (NCCN), St. Gallen, and European Society of Medical Oncology (EMSO) guidelines all proposed that a patient's age should not be considered as a predictive factor of adjuvant chemotherapy but as a prognosis factor [17-19]. Clinicians are still confused about whether additional adjuvant therapy or increased treatment intensity should be rendered for young women. Therefore, big data based on SEER were analyzed in detail to evaluate the impact of age on the outcome in different subgroups. Our aim was to explore the factors that can further stratify the impact of age on the prognosis of breast cancer patients.

\section{Methods}

\section{Study Population}

The SEER database was established by the National Cancer Institute; SEER collects data on patient, disease, and survival outcomes. This national programme includes 18 population-based cancer registries among 14 states across the United States that represents approximately $30 \%$ of the population of the United States. The SEER data do not capture information about surgery and radiation provided past four months of diagnosis, and no information is provided about recurrence or metastasis that is detected subsequent to the initial diagnosis [20]. We obtained the permission to access the research data (Reference Number: 10263-Nov2015). The study was approved by the ethics committee of Zhejiang University Jinhua Hospital. SEER ${ }^{\star S}$ Stat software was used to identify patients diagnosed with breast cancer from January 1990 to December 2007 (Traning cohort). Patients diagnosed after 2007 were excluded to ensure an adequate duration of follow up. The validation data were from 2008 to 2014. The age at diagnosis, marital status, location, race, histological type, stage, survival months and cause of death were retrieved from the SEER database.

The specific inclusion criteria were as follows: (1) site record ICD-O-3 limited to breast cancer (C500-506; C508-509); (2) histological type ICD-O-3 limited to $8500 / 3,8520 / 3,8521 / 3,8522 / 3,8524 / 3$, $8541 / 3$; (3) patients without distant metastases. The exclusion criteria were as follows: (1) patients lacking documentation of race and age at diagnosis; patients younger than 20 years or older than 80 years; (2) patients with multiple primary tumors were excluded to make the analyses of cancer-specific survival more accessible; (3) patients surviving less than one month; and (4) patients with unspecified laterality tumor.

\section{Variable Declaration}

All cases were regrouped according to the 7 th (American Joint Committee on Cancer) AJCC TNM classification system. Race was divided into white, black and others. The hormone-receptor (HoR) status of the tumor was stratified to HoR-positive [estrogen receptor (ER)-positive/progesterone receptor (PR)-positive, ER-negative/PR-positive, ER-positive/ PR-negative] and HoR-(ER-negative/PR-negative). Married status was regrouped as married, single (never married or domestic partner) or divorced ("separated", "divorced", "and widowed"). The age cutoff of 40 years was achieved through the X-tile program (Supplemental data 1) [21].

\section{Statistical Analysis}

The data of all categorical variables were summarized using frequencies and percentages. The X-tile program was used to calculate the cutoff values for age and the risk score [21]. Cancer-specific survival (CSS) was calculated from the date of diagnosis of breast cancer to the date of death from cancer. Death attributed to other causes was defined 
as a censored observation. Survival curves were generated using the Kaplan-Meier method, and the log-rank test was carried out to evaluate the survival differences between groups. The 5 and 10-year CSS rates were calculated using the Life-Table method. Multivariable analyses using Cox regression models with adjusted hazard ratios (HRs) along with 95\% intervals (CIs) were performed to adjust for prognostic variables. Multicollinearity between variables was evaluated, and the variables of stage and HoR were removed in the model. The final multivariate model was chosen based on the stepwise procedure, as well as consideration of the clinical or biologic importance of the variables in the model. Based on the Cox model with identified factors, a nomogram was built to predict the probabilities of CSS at 5 and 10 years. The models were built with race, marital status, location, histological type, differentiated grade, $\mathrm{T}$ classification, $\mathrm{N}$ classification, ER and PR status as variables without the variable of age. The model performance was evaluated in terms of the discrimination and calibration performance. Discrimination is the ability of the predictor to separate patients with different events. Discrimination for survival data was evaluated using the C-index (similar to the area under the receiver operating curve). The C-index ranges from 0 to 1 , with 1 indicating perfect concordance, 0.5 indicating no better concordance than chance, and 0 indicating perfect discordance. In general, the model is considered relatively good to discriminate values above 0.70 . Comparison between the nomogram and current staging systems was performed using the rcorrp.cens function in the Hmisc package in $\mathrm{R}$ and was tested using the $\mathrm{C}$-index. Calibration curves plot the average Kaplan-Meier estimate against the corresponding nomogram for the 5- and 10-year predicted CSS. We used the bootstrapping re-sampling method (100 repetitions) to obtain relatively unbiased estimates and check the interval validation. The probability of breast cancer-specific death in every variable was predicted as a point on the nomogram. The risk score of CSS was calculated for each patient by totalling the points of every variable. Using two optional cutoff values from the $\mathrm{X}$-tile program, the cohort was classified into three subgroups (Supplemental data 2).

When the two-side $P$ value was less than 0.05 , the difference was considered statistically significant. Analyses were performed using statistical software STATA/SE 12.0 (StataCorp LP, TX, USA) and R software (version 3.0.1) with the survival and rms package.

\section{Results}

\section{Patient and tumor characteristics}

We identified 244,324 eligible patients with early breast cancer. The endpoint date of the follow-up was November 2014, with a median follow-up of 110 months (range: 1 to 287 months). In total, 36,826 (15.07\%) patients died of breast cancer. The median age of the patients was 57 years. There were $8.89 \%$ women diagnosed with breast cancer at a younger age. Compared with older patients, younger women were significantly associated with black race (13.08\%), less infiltrative lobular carcinoma $(2.20 \%)$, poorly differentiated tumor $(56.75 \%)$, or late-stage tumor (22\% of stage III tumor). The detailed information is provided in Table 1.

\section{Univariate and multivariate analyses of cancer-specific survival}

Univariate analysis showed that young women with breast cancer had a worse prognosis, with 5- and 10 -year CSS rates of $86.29 \%, 77.48 \%$ and $91.51 \%$, $85.69 \%$ in the younger and older groups, respectively $[\mathrm{HR}=0.62$ (reference to younger group), $P<0.001]$. Additionally, multivariate analysis with variables related to outcomes based on univariate analysis showed that young age was an independent prognostic factor, with $\mathrm{HR}=0.92$ (reference to younger group), $P<0.001$. The detailed information is indicated in Table 2. In subgroup analysis, young women had a worse survival in almost all subgroups, with a significant difference, except for the T4 classification subgroup $(P=0.117)$. The HRs of CSS in the subgroups of N3 classification, poorly differentiated tumor, ER and PR-negative tumor had the trend of lost statistical power. However, we did not identify the rational factor that could further stratify the impact of age on the prognosis of patients with early breast cancer (Fig. 1).

\section{Clinical nomogram prediction and validation of CSS}

A nomogram based on multi-factors, including marital status, race, location, histological type, differentiated grade, $\mathrm{T}$ classification, $\mathrm{N}$ classification, and ER and PR status was constructed (Fig. 2A). Beta-coefficients from the model were used for allocation of scale (Supplemental data 4). The nomogram allows for easy, simultaneous consideration of prognostic factors. $\mathrm{T}$ classification (tumor size and involvement) had the highest impact on CSS. It was unexpected that T4 classification had a greater contribution to survival than N3 classification. Except $\mathrm{T}$ classification and $\mathrm{N}$ classification, differentiated grade was also considered a 
considerable important factor for the outcome. The C-index for the established model was 0.785 (95\% CI: $0.783-0.787)$. The calibration plots indicated an optimal agreement between the nomogram prediction and actual observation for the 2-, 5- and 10-year CSS rates (Fig. 2B).

Table 1: Clinicopathologic characteristics of 244,324 patients with early breast cancer

\begin{tabular}{|c|c|c|c|c|c|c|c|c|c|}
\hline Risk Factors & $\mathrm{N}(\%)$ & $\begin{array}{l}\text { Younger ( } \leq 40 \text { yrs) } \\
N(\%)\end{array}$ & \multicolumn{2}{|l|}{$\mathrm{N}(\%)$} & \multicolumn{5}{|c|}{$\begin{array}{l}\text { well prediction of prognosis with a C-index of } 0.841 \\
\text { (95\% CI: } 0.834-0.848) \text { (Supplemental data } \mathbf{3 A} \text { ). }\end{array}$} \\
\hline Total & 244324 & $21718(8.89)$ & 222606(91.11) & & \multirow{3}{*}{\multicolumn{5}{|c|}{ Table 2: Survival analysis of 244,324 patients with early breast }} \\
\hline Marital status & & & & $<0.001$ & & & & & \\
\hline Married & 154080 & 14806(68.17) & \multicolumn{2}{|l|}{$139274(62.57)$} & & & & & \\
\hline Single & 30907 & $4914(22.63)$ & \multicolumn{2}{|l|}{ 25993(11.68) } & \multicolumn{5}{|c|}{ cancer } \\
\hline Divorce & 59337 & 1998(9.20) & \multicolumn{2}{|l|}{$57339(25.76)$} & & & & & \\
\hline Race & & & & $<0.001$ & \multirow[t]{2}{*}{ Risk Factors } & \multicolumn{2}{|c|}{ Univariate analysis $\mathrm{C}$} & \multicolumn{2}{|c|}{ Multivariate analysis $\mathrm{C}$} \\
\hline White & 202235 & $16474(75.85)$ & \multicolumn{2}{|l|}{$185761(83.45)$} & & $\mathrm{HR}(95 \% \mathrm{CI})$ & $P$ & HR $(95 \%$ CI $)$ & $P$ \\
\hline Black & 21870 & $2840(13.08)$ & \multicolumn{2}{|l|}{$19030(8.55)$} & \multicolumn{5}{|l|}{ Age } \\
\hline Others & 20219 & 2404(11.07) & \multicolumn{2}{|l|}{$17815(8.00)$} & Younger & \multicolumn{2}{|l|}{1} & 1 & \\
\hline Location & & & & 0.148 & Older & $0.62(0.6-0.64)$ & $<0.001$ & $0.92(0.89-0.95)$ & $<0.001$ \\
\hline Left breast & 123960 & $10917(50.27)$ & $113043(50.78)$ & & Marital status & & & & \\
\hline Right breast & 120364 & $10801(49.73)$ & $109563(49.22)$ & & Married & 1 & & 1 & \\
\hline Histological type & & & & $<0.001$ & Single & $1.33(1.29-1.37)$ & $<0.001$ & $1.13(1.1-1.16)$ & $<0.001$ \\
\hline IDC & 208867 & 19995(92.07) & 188872(84.85) & & Divorce & $1.31(1.28-1.34)$ & $<0.001$ & $1.31(1.28-1.35)$ & $<0.001$ \\
\hline ILC & 15338 & $478(2.20)$ & $14860(6.68)$ & & Race & & & & \\
\hline IDC+ILC & 20119 & $1245(5.73)$ & $18874(8.48)$ & & White & 1 & & 1 & \\
\hline Differentiation grade & & & & $<0.001$ & Black & $1.90(1.85-1.96)$ & $<0.001$ & $1.32(1.28-1.36)$ & $<0.001$ \\
\hline Well & 43628 & $1515(6.98)$ & 42113(18.92) & & Others & $0.92(0.89-0.96)$ & $<0.001$ & $0.86(0.83-0.9)$ & $<0.001$ \\
\hline Moderate & 104078 & 7247(33.37) & $96831(43.50)$ & & Location & & & & \\
\hline Poor & 92067 & $12324(56.75)$ & $79743(35.82)$ & & Left breast & 1 & & & \\
\hline Undifferentiated & 4551 & $632(2.91)$ & $3919(1.76)$ & & Right breast & $0.96(0.94-0.98)$ & $<0.001$ & $0.97(0.95-0.99)$ & 0.004 \\
\hline $\mathrm{T}$ Classification A & & & & $<0.001$ & Histological type & & & & \\
\hline $\mathrm{T} 1$ & 153316 & 10467(48.2) & $142849(64.17)$ & & IDC & 1 & & 1 & \\
\hline $\mathrm{T} 2$ & 74287 & 8932(41.13) & $65355(29.36)$ & & ILC & $0.89(0.85-0.93)$ & $<0.001$ & $0.98(0.94-1.03)$ & 0.256 \\
\hline T3 & 11219 & $1761(8.11)$ & $9458(4.25)$ & & IDC+ILC & $0.87(0.84-0.91)$ & $<0.001$ & $0.95(0.91-0.98)$ & 0.004 \\
\hline $\mathrm{T} 4$ & 5502 & $558(2.57)$ & $4944(2.22)$ & & Differentiation & & & & \\
\hline N Classification A & & & & $<0.001$ & grade & & & & \\
\hline No & 155166 & $10817(49.81)$ & $144349(64.85)$ & & Well & 1 & & 1 & \\
\hline N1 & 59933 & $7020(32.32)$ & $52913(23.77)$ & & Moderate & $2.80(2.66-2.94)$ & $<0.001$ & $1.97(1.87-2.07)$ & $<0.001$ \\
\hline N2 & 19027 & $2470(11.37)$ & $16557(7.44)$ & & Poor & $6.08(5.80-6.37)$ & $<0.001$ & $2.74(2.61-2.88)$ & $<0.001$ \\
\hline N3 & 10198 & $1411(6.50)$ & $8787(3.95)$ & & Undifferentiated & $6.20(5.76-6.67)$ & & $2.92(2.71-3.15)$ & $<0.001$ \\
\hline Stage TNM A & & & & $<0.001$ & T Classification A & & & & \\
\hline I & 116511 & $6705(30.87)$ & $109806(49.33)$ & & $\mathrm{T} 1$ & 1 & & 1 & \\
\hline II & 91950 & $10234(47.12)$ & $81716(36.71)$ & & $\mathrm{T} 2$ & $3.26(3.19-3.34)$ & $<0.001$ & $1.92(1.87-1.97)$ & $<0.001$ \\
\hline III & 35863 & $4779(22)$ & $31084(13.96)$ & & T3 & $5.66(5.46-5.87)$ & $<0.001$ & $2.44(2.35-2.54)$ & $<0.001$ \\
\hline ER & & & & $<0.001$ & $\mathrm{~T} 4$ & $10.56(10.14-11)$ & $<0.001$ & $3.93(3.76-4.11)$ & $<0.001$ \\
\hline Negative & 56257 & $7998(36.83)$ & $48259(21.68)$ & & N Classification A & & & & \\
\hline Positive & 188067 & $13720(63.17)$ & $174347(78.32)$ & & No & 1 & & 1 & \\
\hline PR & & & & $<0.001$ & N1 & $2.56(2.49-2.62)$ & $<0.001$ & $1.97(1.92-2.02)$ & $<0.001$ \\
\hline Negative & 80849 & $9158(42.17)$ & $71691(32.21)$ & & $\mathrm{N} 2$ & $5.4(5.24-5.56)$ & $<0.001$ & $3.32(3.21-3.42)$ & $<0.001$ \\
\hline Positive & 163475 & $12560(57.83)$ & $150915(67.79)$ & & N3 & $9.66(9.36-9.97)$ & $<0.001$ & $5.23(5.05-5.41)$ & $<0.001$ \\
\hline HoR & & & & $<0.001$ & Stage TNM A & & & & \\
\hline Negative & 51230 & $7182(33.07)$ & $44048(19.79)$ & & I & 1 & & $(-)$ & \\
\hline Positive & 193094 & $14536(66.93)$ & $178558(80.21)$ & & II & $3.18(3.09-3.27)$ & $<0.001$ & $(-)$ & \\
\hline Risk Groups B & & & & $<0.001$ & III & $9.70(9.43-9.99)$ & $<0.001$ & $(-)$ & \\
\hline Low & 134953 & $7656(35.25)$ & 127297(57.18) & & ER & & & & \\
\hline Medium & 82996 & $10100(46.51)$ & $72896(32.75)$ & & Negative & 1 & & 1 & \\
\hline High & 26375 & $3962(18.24)$ & $22413(10.07)$ & & Positive & $0.45(0.44-0.46)$ & $<0.001$ & $0.79(0.76-0.81)$ & $<0.001$ \\
\hline $\begin{array}{l}\text { A Stage TNM T/N clas } \\
\text { system. }\end{array}$ & ication ac & cording to the $7^{\text {th }}$ ed & on of the AJCC s & ging & $\begin{array}{l}\text { PR } \\
\text { Negative }\end{array}$ & 1 & & 1 & \\
\hline $\begin{array}{l}\text { B The probability of bre } \\
\text { point on the nomogral }\end{array}$ & $\begin{array}{l}\text { St cancer- } \\
\text { The risk }\end{array}$ & $\begin{array}{l}\text { pecific death in ever } \\
\text { score of CSS was calc }\end{array}$ & $\begin{array}{l}\text { variable was pre } \\
\text { lated for each pa }\end{array}$ & $\begin{array}{l}\text { icted as a } \\
\text { ent by }\end{array}$ & $\begin{array}{l}\text { Positive } \\
\text { HoR }\end{array}$ & $0.48(0.47-0.49)$ & $<0.001$ & $0.77(0.75-0.79)$ & $<0.001$ \\
\hline summing the points of & very vari & ble. Using two cuto & values from the $>$ & & Negative & 1 & & $(-)$ & \\
\hline program, the cohort $\mathrm{w}$ & classifiec & into three risk grou & & & Positive & $0.45(0.44-0.46)$ & $<0.001$ & $(-)$ & \\
\hline c $P$ values obtained fro & $\chi^{2}$ test. $A$ & ll statistical tests we & two-sided. & & Risk Groups ${ }^{\text {B }}$ & & & & \\
\hline Abbreviations: $\mathrm{HoR}=\mathrm{H}$ & rmone re & ceptor; $\mathrm{ER}=$ Estrogen & eceptor; PR= Pro & esterone & Low & 1 & & $(-)$ & \\
\hline receptor; IDC= Infiltra & ag duct ce & rcinoma; ILC= Infilt & ting lobular carci & & Medium & $3.98(3.88-4.09)$ & $<0.001$ & $(-)$ & \\
\hline & & & & & High & $12.53(12.18-12.89)$ & $<0.001$ & $(-)$ & \\
\hline $\begin{array}{l}\text { Based on } \\
\text { into three }\end{array}$ & $\begin{array}{l}\text { he risk } \\
\text { group }\end{array}$ & $\begin{array}{l}\text { score, the co } \\
\text { s (classified }\end{array}$ & $\begin{array}{l}\text { ort was cla } \\
\text { by X-tile }\end{array}$ & $\begin{array}{r}\text { sified } \\
\text { low }\end{array}$ & $\begin{array}{l}\text { A Stage TNM T/N } \\
\text { system. }\end{array}$ & classification accor & ding to $t$ & edition of the A & taging \\
\hline
\end{tabular}

CCSD-RS: 0-109, medium CCSD-RS: 110-186 and high CCSD-RS: $>186$. Univariate analysis showed the risk score was associated with the prognosis, with 5- and 10 -year CSS rates of $97.8 \%$ and $94.6 \%$ in the low-risk subgroup, $88.1 \%$ and $78.9 \%$ in the medium-risk subgroup, and $63.3 \%$ and $49.9 \%$ in the high-risk subgroup, respectively (Fig. 3). The model was also verified by the 2008-2014 validation cohort. In the validation cohort, the nomogram risk model showed a well prediction of prognosis with a C-index of 0.841

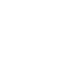


в The probability of breast cancer-specific death in every variable was predicted as a point on the nomogram. The risk score of CSS was calculated for each patient by summing the points of every variable. Using two cutoff values from the X-tile program, the cohort was classified into three risk groups.

c Univariate and multivariate analyses were conducted using the Cox proportional hazards regression model.

Abbreviations: $\mathrm{HoR}=$ Hormone receptor; $\mathrm{ER}=$ Estrogen receptor; $\mathrm{PR}=$ Progesterone receptor; IDC= Infiltrating duct carcinoma; ILC= Infiltrating lobular carcinoma.

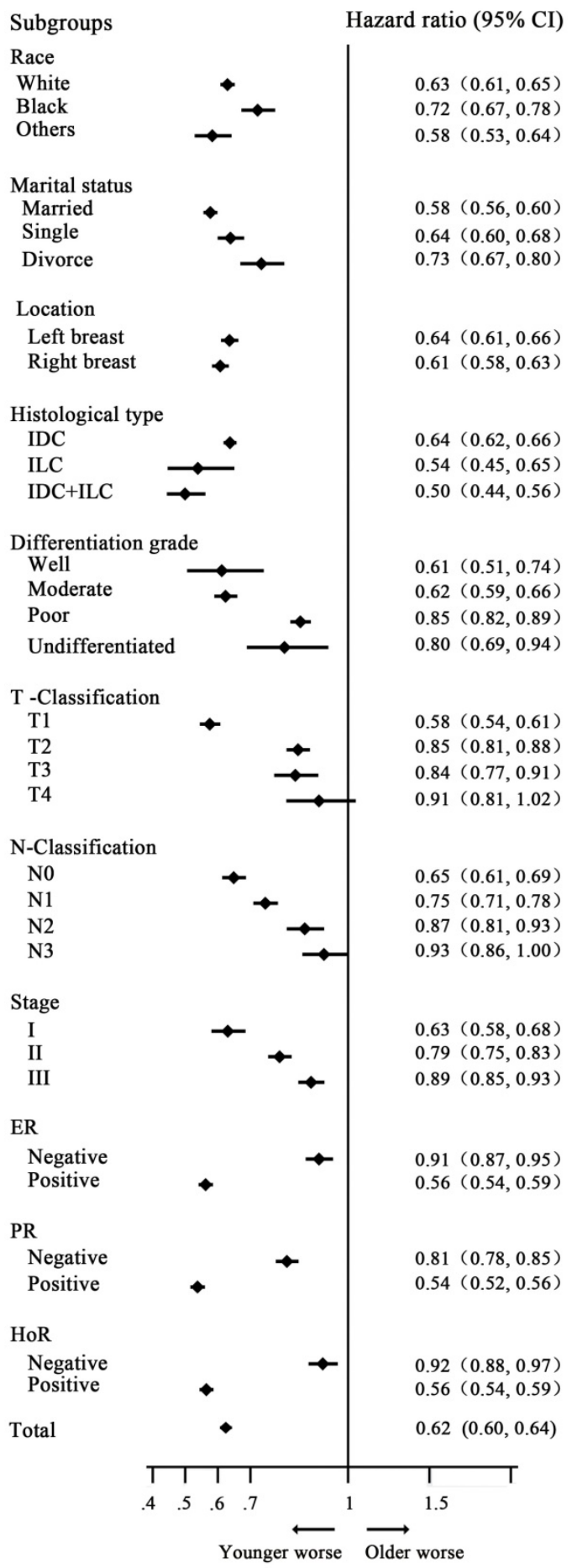

Fig. 1: Stratified analysis by variable and probability of breast cancer-specific survival analysis by age. Young women with breast cancer had a worse survival in almost all subgroups, with a significant difference, except for the T4 subgroup $(P=0.117)$.

\section{Impact of age on cancer-specific survival following stratification analysis by the risk score}

In the low-risk subgroup, older women with breast cancer showed a 39\% decreased probability of death caused by breast cancer $(\mathrm{HR}=0.61 ; 95 \% \mathrm{CI}$ : 0.57-0.65). In the medium-risk subgroup, older women with breast cancer showed only a $10 \%$ decreased probability of death caused by breast cancer (HR=0.89; 95\% CI: 0.85-0.93). However, in the high-risk subgroup, younger patients had a similar prognosis to that of older patients $(P=0.431)$ (Fig. 4). As shown in the Kaplan-Meier curve of Figure $4 C$, the two survival curves had a tendency to separate from each other with a prolonged follow-up time, indicating that the protective role of young age in high-risk breast cancer became more obvious.

Additionally, we further performed external validation (2008-2014). Because the sample size was inadequate (small proportion of young patients), we combined the low- and medium-risk subgroups. The results showed a similar outcome in the training cohort (1990-2007). Young age did not act as a prognostic factor in high risk group (Supplemental data 3B).

\section{Discussion}

The accurate prediction of survival is an essential part of the decision-making process following surgery for early breast cancer and allows clinicians to determine which patients will benefit from adjuvant therapy. A computer-based, validated model, Adjuvant! online, is a widely used model in clinical practice. The model was based on the 10-year observed overall survival of women aged 36 to 69 years who were diagnosed between 1988 and 1992 and were recorded in SEER [22]. The age group 36 to 69 years was selected to eliminate bias considering that women younger than 35 years had a worse prognosis than those older than 35 years. Another important tool is PREDICT [23], which is based on information from 5,694 women with breast cancer in England. The Cox proportional hazard model indicated that age as a categorical variable in five age groups ( $<40,40$ to 49,50 to 59,60 to 69 and $>70$ years) was not an independent factor to predict CSS in their cohort. Considering that older patients were associated with more death from other causes, age was set to the power of 2.38 in their model.

In the current study, analysis based on big data showed that age was an independent prognostic factor. Subgroup analysis also showed that young breast cancer had a worse prognosis regardless of subgroup. However, the poorer prognosis subgroups (T4 classification, N3 classification, poorly 
differentiated tumor, ER-negative tumor, PR-negative tumor) showed a weaker association between young age and survival; young age will lose prognostic significance with an increasing risk of breast cancer-specific death. Thus, age was excluded in our nomogram. Meanwhile, the risk score yielded from nomogram points was utilized to stratify the impact of young age on the prognosis. To our knowledge, we first constructed a risk model without age to explore the real impact of age on the survival of breast cancer patients.

The variable of age, as a prognostic factor, has been controversial for some time [8, 24-27]. Stratification analysis based on the molecular subtype

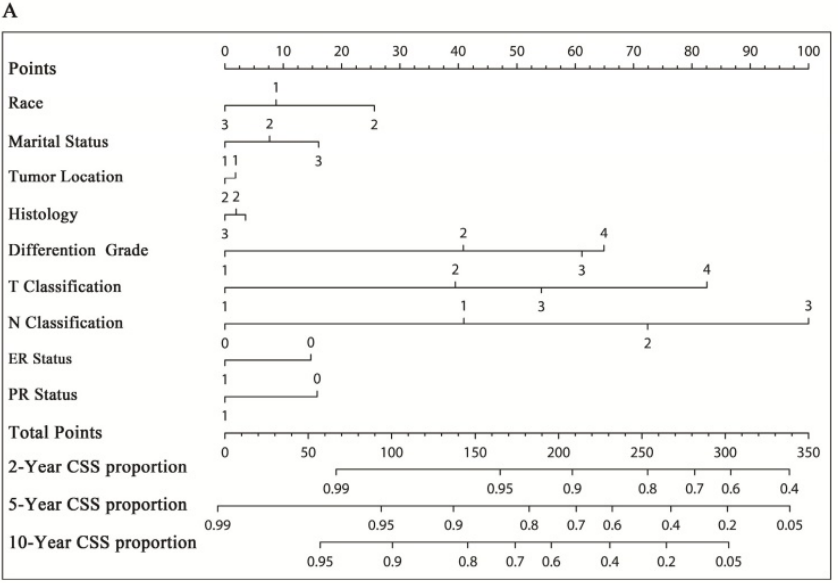

showed that younger breast cancer was more common with the triple-negative subtype or HER2 over-expression subtype than luminal breast cancer $[11,28]$. Further prognostic analysis indicated that a worse prognosis of the younger group was associated with the luminal subtype rather than the triple-negative subtype or HER2 over-expression subtype [9]. However, Cancello et al[28] revealed an opposite result in that younger patients had a similar prognosis to that of older patients with the luminal A subtype (ER-positive/PR-positive) of breast cancer and had a poor prognosis compared with older patients with the luminal B subtype, triple-negative subtype and HER2 over-expression subtype.

B

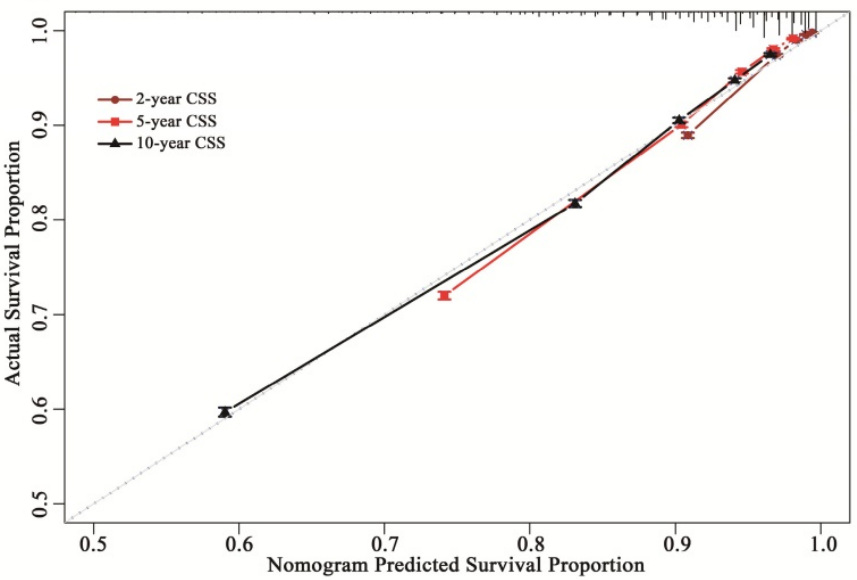

Fig. 2. A: Nomogram to predict cancer-specific survival (CSS) in early breast cancer. The nomogram is used by summing the points identified on the top scale for each independent covariate. The total points projected to the bottom scale indicate the \% probability of the 2-, 5-, and 10-year CSS. Race: 1=white, 2=black, 3=other; Histological type: $1=$ infiltrative duct cancer, $2=$ infiltrative lobular cancer, $3=$ Infiltrating duct and lobular carcinoma. Tumor location: $1=$ left, $2=$ right; $\mathrm{T}$ and $\mathrm{N}$ classification according to the 7th AJCC TNM system. ER= estrogen receptor: $1=$ positive, $0=$ negative. $P R=$ progesterone receptor: $1=$ positive, $0=$ negative. Marital status: $1=$ married; $2=$ single (never married or domestic partner), 3 = divorced (separated, divorced, widowed). B: Calibration curve for predicting patient cancer-specific survival (CSS) at 5 and 10 years. The nomogram-predicted probability of CSS is plotted on the $\mathrm{x}$-axis; the actual CSS is plotted on the $y$-axis. CSS= cancer-specific survival

\section{Cancer Specific Survival}

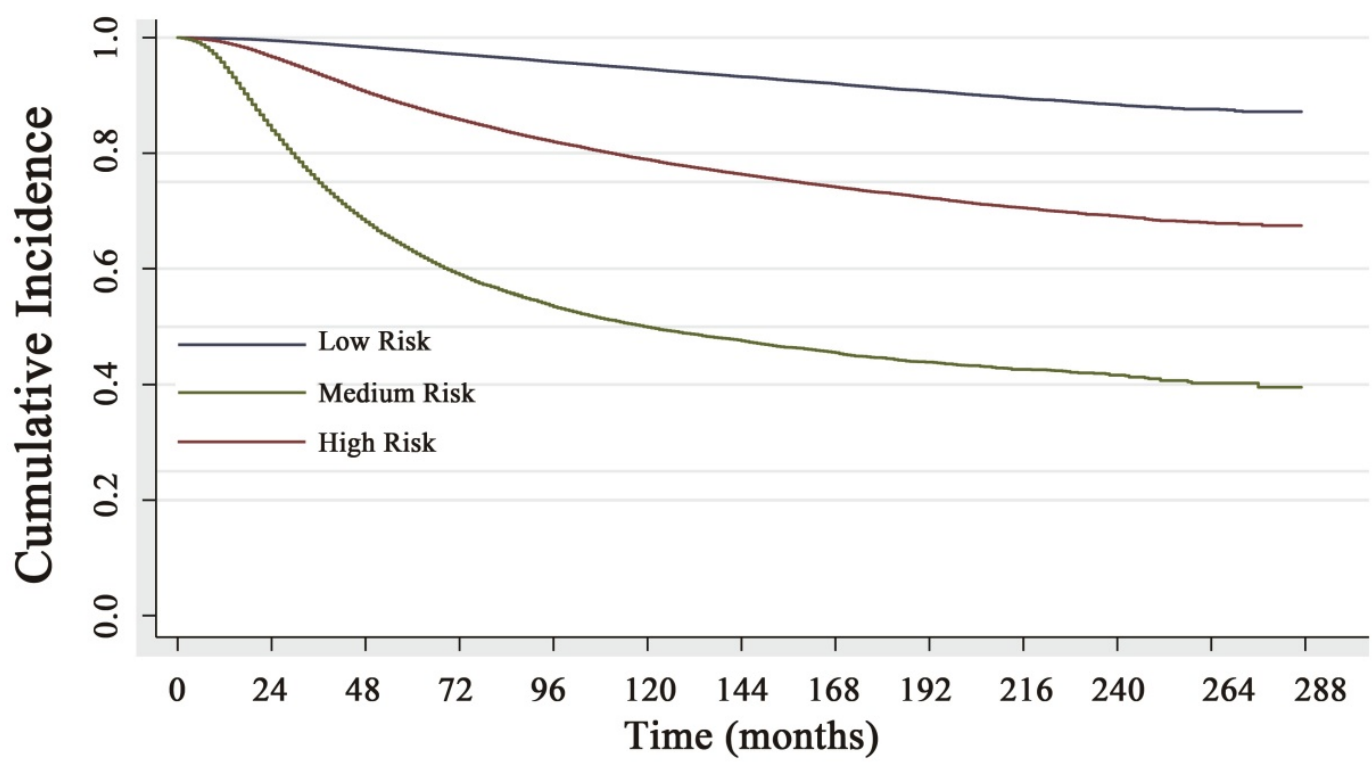

Fig. 3: Cancer-specific survival (CSS) curve of the low-risk, medium-risk and high-risk groups. The 5 - and 10 -year CSS rates of $97.8 \%$ and $94.6 \%$ in the low-risk subgroup, $88.1 \%$ and $78.9 \%$ in the medium-risk subgroup, and $63.3 \%$ and $49.9 \%$ in the high-risk subgroup, respectively, with a significant difference $(P<0.001)$. 

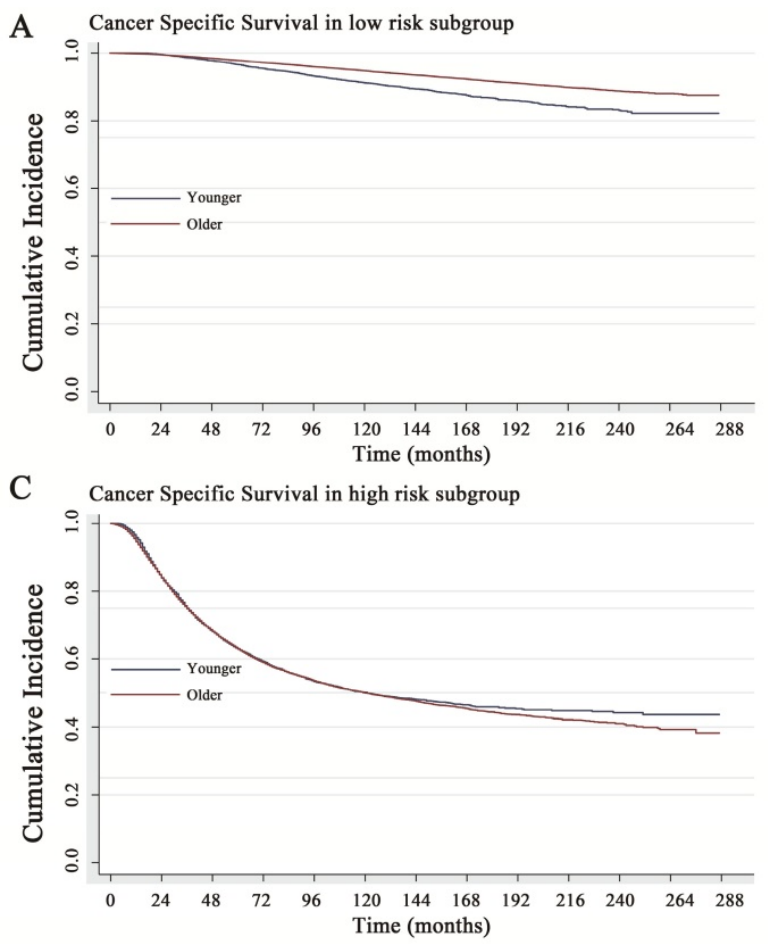

B

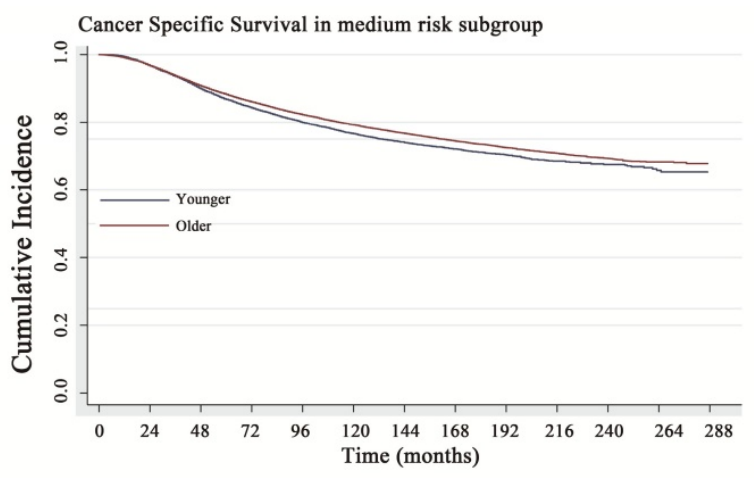

D

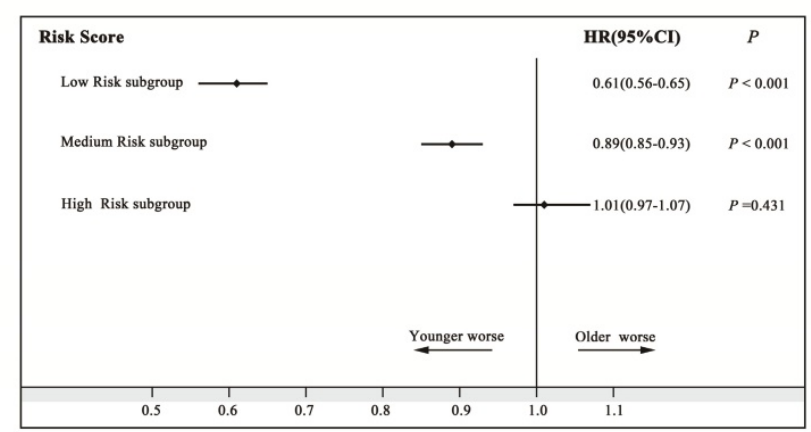

Fig. 4: Impact of age on cancer-specific survival with stratification analysis by the risk score. A: In the low-risk subgroup, the older women with breast cancer showed a $39 \%$ decreased probability of death caused by breast ( $\mathrm{HR}=0.61 ; 95 \% \mathrm{Cl}: 0.57-0.65)$. $\mathrm{B}$ : In the medium-risk subgroup, the older women with breast cancer showed only a $10 \%$ decreased probability of death caused by breast cancer $(\mathrm{HR}=0.89 ; 95 \% \mathrm{Cl}: 0.85-0.93)$. C: In the high-risk subgroup, young age acted as a favorable factor, and older women with breast cancer showed a $2 \%$ increased probability of death caused by breast cancer, without statistical significance (HR=1.02; $95 \% \mathrm{Cl}$ : $0.97-1.07)$, $(P=0.431)$. $D$ : Forest plot of stratification analysis by the risk score for the probability of breast cancer-specific death in younger and older women with breast cancer. HR: hazard ratio

Additionally, Kim et al. revealed that young patients with the luminal subtype and HER2 over-expression subtype had a poor prognosis. Furthermore, some studies showed that age could impact the prognosis regardless of the intrinsic subtype [12]. Our current study also showed that young patients had a worse prognosis than old patients regardless of the ER and PR status.

More importantly, our study indicated that the prognostic value of young age was significantly associated with the risk status. In the low-risk subgroup, young breast cancer had worse prognosis than older breast cancer. However, in the high-risk subgroup, young age acted as a prognostic protective factor. The issue of whether an extra benefit of adjuvant chemotherapy will be expected in low-risk young breast cancer. SOFT (Suppression of Ovarian Function Trial) and TEXT (Tamoxifen and Exemestane Trial) trials [29] suggested that premenopausal women with ER positive and medium/high, as defined by clinicopathologic characteristics risk, might experience survival improvement with more aggressive antihomonal treatment may benefit, while the improvement was minimal for those at low risk. However, our data showed that age was still an important issue to be considered for patients at low risk. As young patients had obviously poorer prognosis in low risk group, more aggressive antihomonal treatment should be considered for young patients with low risk. Additionally, regarding low-risk patients, the 21-gene expression assay (Oncotype Dx recurrence score) would be recommended in clinical practice [14]. Using gene signatures to predict the prognosis and guide the individual treatment does not conflict with our strategy. We used traditional clinicopathological factors to construct the model in which genomic signatures can compensate for each other. The Oncotype Dx recurrence score is widely used to evaluate the likelihood of distant recurrence in breast cancer patients with ER-positive and lymph node-negative infiltration and the benefit of adjuvant chemotherapy. Patients with ER-positive and lymph node-negative breast cancer had more probabilities in the low-risk subgroup with a low-risk score (the correlation of ER-positive and lymph node-negative breast cancer with a low risk was not shown in the current study). The current study showed that young breast cancer had a worse outcome in the low-/medium-risk group. Thus, if young age and low-risk subgroup patients received the 21-gene expression assay, more patients should obtain the high recurrence score (recurrence score $>35$ ). In Paik's study, 33/59 (56\%) young patients (< 40 years) were identified as having a high recurrence score, while the rate in older patients (>60 years) was only $21 \%$ 
(64/310) [14]. Our hypothesis was consistent with their results. Furthermore, the MammaPrint system also confirmed our hypnosis; 52/63(82\%) young patients were classified as high risk in MammaPrint [13]. If young age was proven to be a stronger independent prognostic factor than that for the low-risk subgroup, the application of such a gene signature system will be weakened considering the cost effectiveness.

In traditional opinion, young age is always associated with a poor prognosis. In the high-risk subgroup, additional therapy (intensive multi-agent regimens) will be selected because of young age, inevitably resulting in overtreatment because our current study showed that young age may act as a protective factor of prognosis. This opinion was also consistent with the ESMO Clinical Practice Guidelines that "young" patients should not be over-treated because they are "young" [18]. Additionally, Adjuvant! online or the PREDICT system cannot predict the prognosis of younger or the youngest women with breast cancer. Nearly $10 \%$ of young women will die of breast cancer, even those in the low-risk subgroup. Additionally, young patients with breast cancer will likely obtain a high recurrence score after being subjected to an expensive gene signature array. The recommendation that "young" should no longer be considered a predictive factor of adjuvant chemotherapy may only be suitable for the mediumor high-risk subgroup rather than for the young low-risk subgroup.

Our study possesses several potential limitations. Some information on some other prognostic factors, such the HER2 status and Ki67 in the SEER system was not provided before 2010. Additionally, the intrinsic subtype could not be analyzed. Furthermore, the information on adjuvant therapy is not available in the SEER database, impacting the outcome. The data on tumor recurrence were not provided; thus, the disease-free survival was not available. Additionally, the relationship between the risk score and administration of chemotherapy could not be analyzed.

\section{Conclusion}

The worse prognosis of young women only appeared in the low- and medium-risk subgroups rather than in the high-risk subgroup. The risk score yielded from the nomogram model can assist clinical decision making for young breast cancer patients.

\section{Abbreviations} Results.

SEER: Surveillance, Epidemiology, and End

CSS: Cancer-specific survival.
HR: Hazard ratio.

CI: Confidence intervals.

ER: Estrogen receptor.

HER2: Human epidermal growth factor receptor

2

PR: Progesterone receptor.

HoR: Hormone receptor.

AJCC: American Joint Committee on Cancer.

NCCN: National Comprehensive Cancer

Network.

EMSO: European Society of Medical Oncology.

BRCA: breast cancer susceptibility gene.

SOFT: Suppression of Ovarian Function Trial.

TEXT: Tamoxifen and Exemestane Trial.

\section{Supplementary Material}

Supplementary data.

http://www.jcancer.org/v10p3124s1.pdf

\section{Acknowledgements}

\section{Funding}

The study was partially supported by Natural Science Foundation of Zhejiang Province in China (LGF18H160029 and 2014-3-008) and key program of the Jinhua Municipal Science \& Technology Bureau (2016-3-005). The work was sponsored by the ZhengShu Medical Elite Scholarship Fund.

\section{Ethical approval}

The study was approved by the ethics committee of Zhejiang University Jinhua Hospital.

\section{Informed consent}

Informed consent was obtained from the SEER database whose data are included in this article.

\section{Author Contributions}

Conception and design: Jinin $\mathrm{Du}$, Jianfei Fu and Lunpo $\mathrm{Wu}$.

Data collection analysis and interpretation: Jianfei $\mathrm{Fu}$ and Lunpo Wu.

Statistical review: Wei Fu.

Manuscript writing: All authors.

Final approval of manuscript: All authors.

\section{Competing Interests}

The authors have declared that no competing interest exists.

\section{References}

1. Ribnikar D, Ribeiro JM, Pinto D, Sousa B, Pinto AC, Gomes E, et al. Breast cancer under age 40: a different approach. Current treatment options in oncology. 2015; 16: 16

2. Goldhirsch A, Wood WC, Gelber RD, Coates AS, Thurlimann B, Senn HJ, et al. Progress and promise: highlights of the international expert consensus on the primary therapy of early breast cancer 2007. Ann Oncol. 2007; 18: 1133-44. 
3. Eifel P, Axelson JA, Costa J, Crowley J, Curran WJ, Jr., Deshler A, et al. National Institutes of Health Consensus Development Conference Statement: adjuvant therapy for breast cancer, November 1-3, 2000. Journal of the National Cancer Institute. 2001; 93: 979-89.

4. Freedman RA, Partridge AH. Management of breast cancer in very young women. Breast (Edinburgh, Scotland). 2013; 22 Suppl 2: S176-9.

5. Cardoso F, Loibl S, Pagani O, Graziottin A, Panizza P, Martincich L, et al. The European Society of Breast Cancer Specialists recommendations for the management of young women with breast cancer. European journal of cancer (Oxford, England : 1990). 2012; 48: 3355-77.

6. Ahn SH, Son BH, Kim SW, Kim SI, Jeong J, Ko SS, et al. Poor outcome of hormone receptor-positive breast cancer at very young age is due to tamoxifen resistance: nationwide survival data in Korea--a report from the Korean Breast Cancer Society. Journal of clinical oncology : official journal of the American Society of Clinical Oncology. 2007; 25: 2360-8.

7. Han W, Kang SY, Korean Breast Cancer S. Relationship between age at diagnosis and outcome of premenopausal breast cancer: age less than 35 years is a reasonable cut-off for defining young age-onset breast cancer. Breast Cancer Res Treat. 2010; 119: 193-200

8. Kothari AS, Beechey-Newman N, D'Arrigo C, Hanby AM, Ryder K, Hamed H, et al. Breast carcinoma in women age 25 years or less. Cancer. 2002; 94: 606-14.

9. Sheridan W, Scott T, Caroline S, Yvonne Z, Vanessa B, David V, et al. Breast cancer in young women: have the prognostic implications of breast cancer subtypes changed over time? Breast cancer research and treatment. 2014; 147: $617-29$

10. El Saghir NS, Seoud M, Khalil MK, Charafeddine M, Salem ZK, Geara FB, et al. Effects of young age at presentation on survival in breast cancer. BMC cancer. 2006; 6: 194

11. Kim HJ, Han W, Yi OV, Shin HC, Ahn SK, Koh BS, et al. Young age is associated with ipsilateral breast tumor recurrence after breast conserving surgery and radiation therapy in patients with HER2-positive/ER-negative subtype. Breast cancer research and treatment. 2011; 130: 499-505.

12. Azim HA, Jr., Michiels S, Bedard PL, Singhal SK, Criscitiello C, Ignatiadis M, et al. Elucidating prognosis and biology of breast cancer arising in young women using gene expression profiling. Clinical cancer research : an official journal of the American Association for Cancer Research. 2012; 18: 1341-51.

13. van de Vijver MJ, He YD, van't Veer LJ, Dai H, Hart AA, Voskuil DW, et al. A gene-expression signature as a predictor of survival in breast cancer. The New England journal of medicine. 2002; 347: 1999-2009.

14. Paik S, Shak S, Tang G, Kim C, Baker J, Cronin M, et al. A multigene assay to predict recurrence of tamoxifen-treated, node-negative breast cancer. The New England journal of medicine. 2004; 351: 2817-26.

15. Anders CK, Hsu DS, Broadwater G, Acharya CR, Foekens JA, Zhang Y, et al. Young age at diagnosis correlates with worse prognosis and defines a subset of breast cancers with shared patterns of gene expression. Journal of clinical oncology : official journal of the American Society of Clinical Oncology. 2008; 26: $3324-30$

16. Anders CK, Fan C, Parker JS, Carey LA, Blackwell KL, Klauber-DeMore N, et al. Breast carcinomas arising at a young age: unique biology or a surrogate for aggressive intrinsic subtypes? Journal of clinical oncology : official journal of the American Society of Clinical Oncology. 2011; 29: e18-20.

17. Senkus E, Kyriakides S, Ohno S, Penault-Llorca F, Poortmans P, Rutgers E, et al. Primary breast cancer: ESMO Clinical Practice Guidelines for diagnosis, treatment and follow-up. Annals of oncology : official journal of the European Society for Medical Oncology / ESMO . 2015; 26 Suppl 5: v8-30.

18. Zagouri F, Liakou P, Bartsch R, Peccatori FA, Tsigginou A, Dimitrakakis C, et al. Discrepancies between ESMO and NCCN breast cancer guidelines: An appraisal. Breast (Edinburgh, Scotland). 2015; 24: 513-23.

19. Coates AS, Winer EP, Goldhirsch A, Gelber RD, Gnant M, Piccart-Gebhart M, et al. Tailoring therapies--improving the management of early breast cancer: St Gallen International Expert Consensus on the Primary Therapy of Early Breast Cancer 2015. Annals of oncology : official journal of the European Society for Medical Oncology. 2015; 26: 1533-46.

20. McCammon R, Finlayson C, Schwer A, Rabinovitch R. Impact of postmastectomy radiotherapy in T3N0 invasive carcinoma of the breast: a Surveillance, Epidemiology, and End Results database analysis. Cancer. 2008; 113: 683-9.

21. Camp RL, Dolled-Filhart M, Rimm DL. X-tile: a new bio-informatics tool for biomarker assessment and outcome-based cut-point optimization. Clin Cancer Res. 2004; 10: 7252-9.

22. Ravdin PM, Siminoff LA, Davis GJ, Mercer MB, Hewlett J, Gerson N, et al. Computer program to assist in making decisions about adjuvant therapy for women with early breast cancer. Journal of clinical oncology : official journal of the American Society of Clinical Oncology. 2001; 19: 980-91.

23. Wishart GC, Azzato EM, Greenberg DC, Rashbass I, Kearins O, Lawrence G, et al. PREDICT: a new UK prognostic model that predicts survival following surgery for invasive breast cancer. Breast cancer research : BCR. 2010; 12: R1.

24. Rapiti E, Fioretta G, Verkooijen HM, Vlastos G, Schafer P, Sappino AP, et al. Survival of young and older breast cancer patients in Geneva from 1990 to 2001. European journal of cancer (Oxford, England : 1990). 2005; 41: 1446-52.

25. Barchielli A, Balzi D. Age at diagnosis, extent of disease and breast cancer survival: a population-based study in Florence, Italy. Tumori. 2000; 86: 119-23.

26. La Rosa F, Patavino VM, Epifani AC, Petrinelli AM, Minelli L, Mastrandrea V. Ten-year survival and age at diagnosis of women with breast cancer from a population-based study in Umbria, Italy. Tumori. 1996; 82: 441-3.
27. Kheirelseid EH, Boggs JM, Curran C, Glynn RW, Dooley C, Sweeney KJ, et al. Younger age as a prognostic indicator in breast cancer: a cohort study. BMC cancer. 2011; 11: 383

28. Cancello G, Maisonneuve P, Rotmensz N, Viale G, Mastropasqua MG, Pruneri $G$, et al. Prognosis and adjuvant treatment effects in selected breast cancer subtypes of very young women ( $<35$ years) with operable breast cancer. Annals of oncology : official journal of the European Society for Medical Oncology. 2010; 21: 1974-81.

29. Regan MM, Francis PA, Pagani O, Fleming GF, Walley BA, Viale G, et al. Absolute Benefit of Adjuvant Endocrine Therapies for Premenopausal Women With Hormone Receptor-Positive, Human Epidermal Growth Factor Receptor 2-Negative Early Breast Cancer: TEXT and SOFT Trials. J Clin Oncol. 2016; 34: 2221-31 